\title{
The conformal boundary of anti-de Sitter space-times
}

\author{
Charles Frances
}

May 26, 2011

\section{Introduction}

Given a non compact space-time $(M, g)$ (i.e a manifold $M$ endowed with a Lorentzian metric $g$ ), the study of the geometrical asymptotic properties of $M$ is of great mathematical, as well as physical interest. This kind of study was pioneered by R. Penrose (see $[\mathrm{P}]$ ), by associating a conformal boundary to some space-times. More precisely, a conformal boundary completion of a space-time $(M, g)$, is a manifold $\bar{M}$, with boundary $\partial M$, endowed with a conformal class of Lorentzian metrics $[\bar{g}]$, satisfying the following conditions:

(i) The interior of $(\bar{M},[\bar{g}])$ is conformally diffeomorphic to $(M, g)$.

(ii) For a metric $\bar{g}$ in the conformal class $[\bar{g}]$, there is a smooth function $\rho$ on $\bar{M}$, such that $\rho^{-1}(\{0\})=\partial M, d \rho \neq 0$ on $\partial M$, and $\bar{g}=\rho^{2} g$ on $M$.

Notice that if $\bar{g} \in[\bar{g}]$, the causal caracter of $\bar{g}_{\mid \partial M}$ can be non constant on $\partial M$. For example, if $B$ is an open (Euclidian) ball in Minkowski space, the Minkowski metric restricted to $\partial B$ is Lorentzian at some points, Riemannian or degenerate at others.

In $[\mathrm{P}]$, Penrose constructed a conformal boundary completion for several families of space-times, but in the general case, two natural questions remain widely open: under which minimal hypotheses does such a conformal boundary completion exist? When it exists, do we have some kind of uniqueness result?

In the Riemannian context, a lot of works concerning conformally compact Einstein metrics where done (see $[\mathrm{A}],[\mathrm{C}-\mathrm{H}],[\mathrm{Bi}]$ and many others), and some partial results are known about the two previous questions.

The case of compact conformal boundary completions (or conformal compactifications) is not the only relevant. In fact, very basic and natural space-times involve non compact conformal boundary completions. One example, which will be fundamental in what follows, is anti-de Sitter space $\widetilde{\mathbf{A d S}}_{n+1}$ (the simply connected complete Lorentzian manifold of curvature -1). This space admits as conformal boundary Einstein's static universe $\widetilde{\operatorname{Ein}}_{n}$ (see section 2), which is topologically $\mathbf{R} \times \mathbf{S}^{n-1}$. Building a conformal 
boundary completion of other anti-de Sitter space-times (i.e lorentzian manifolds of constant curvature -1) can be also usefull, as shows the example of BTZ blackholes (see [BTZ1],[BTZ2]). These blackholes are particular cases of Kleinian anti-de Sitter structures, namely the quotient of some open subset $\Omega \subset \widetilde{\mathbf{A d S}}_{n+1}$ by a discrete subgroup of isometries $\Gamma \subset \widetilde{O(2, n)}$. In this paper, we will focus on a very special kind of Kleinian structures, namely the complete anti-de Sitter structures. In other words, we will deal mainly with space-times of the form $\widetilde{\mathbf{A d S}}_{n+1} / \Gamma$, for a discrete $\Gamma \subset \widetilde{O(2, n)}$ acting properly discontinuously on $\widetilde{\mathbf{A d S}}_{n+1}$. The aim of this article is to build a conformal boundary completion for these complete structures. We will also get a uniqueness result (Theorem 1), and prove that in this context, the conformal structure on the boundary $\left(\partial M,[\bar{g}]_{\mid \partial M}\right)$ determines completely $(M, g)$ (Theorem 2). In section 5, we give an illustration of these constructions on explicit examples.

Notice that the restriction to the case of complete structures is not "physically realistic". Indeed, to get space-times without causality pathologies (such as closed causal geodesics) it is in general necessary to consider only quotients of strict open subsets of $\widetilde{\mathbf{A d S}}_{n+1}$ (as it is the case for BTZ blackholes). Nevertheless, we think that the methods presented here will be usefull to deal with the general case of Kleinian structures.

\section{Anti-de Sitter space and Einstein's universe}

We recall here some basic properties of Einstein's universe and anti-de Sitter space. we refer to $[\mathrm{HE}],\left[\mathrm{O}^{\prime} \mathrm{N}\right],[\mathrm{S}]$ and $[\mathrm{Fr} 1]$ for more details.

\subsection{Einstein's static universe}

Let $\mathbf{R}^{2, n+1}$ be the space $\mathbf{R}^{n+3}$, endowed with the quadratic form $q^{2, n+1}(x)=$ $-2 x_{1} x_{n+3}-2 x_{2} x_{n+2}+x_{3}^{2}+\ldots+x_{n}^{2}+x_{n+1}^{2}$. We call $C^{2, n+1}$ the isotropic cone of $q^{2, n+1}$. The projection of $C^{2, n+1}$ on $\mathbf{R} P^{n+2}$ is a smooth quadric, endowed with a natural Lorentzian conformal structure inherited from $\mathbf{R}^{2, n+1}$. This quadric, together with its natural structure is called the compact Einstein's universe of dimension $n+1$, and denoted $\operatorname{Ein}_{n+1}$. The group $P O(2, n+1)$ acts conformally on $\mathbf{E i n}_{n+1}$ and in fact, this is exactly the conformal group of $\mathbf{E i n}_{n+1}$. The space $\mathbf{E i n}_{n+1}$ is not simply connected and admits $\mathbf{R} \times \mathbf{S}^{n}$ as universal cover. The lift of the conformal class of $\mathbf{E i n}_{n+1}$ is the conformal class of the metric $-d t^{2}+g_{S^{n}}$. The pair $\left(\mathbf{R} \times \mathbf{S}^{n},\left[-d t^{2}+g_{S^{n}}\right]\right)$ is called static Einstein's universe, and denoted $\widetilde{\operatorname{Ein}}_{n+1}$. Any conformal transformation of $\operatorname{Ein}_{n}$ can be lifted into a conformal transformation of $\widetilde{\mathbf{E i n}}_{n}$, and we denote by $O(\widetilde{2, n+1})$ the lifting of the whole Lorentzian Möbius group $O(2, n+1)$. The identity component $S O^{\circ}(2, n+1)$ has a center, isomorphic to $\mathbb{Z}$ and 
generated by $\zeta:(t, x) \mapsto(t+\pi,-x)$. The space $\operatorname{Ein}_{n+1}$ is just the quotient of $\widetilde{\operatorname{Ein}}_{n+1}$ by the action of the center.

\section{$2.2 \quad$ Lightlike geodesics}

It is well known that in pseudo-Riemannian conformal geometry, lightlike geodesics have a conformal meaning. Indeed, all the metrics of a same conformal class have the same lightlike geodesics (if one forgets the parametrization). On $\widetilde{\mathbf{E i n}}_{n+1}$, lightlike geodesics admit a parametrization of the form: $t \mapsto(t, c(t))$, where $t \mapsto c(t)$ is a geodesic of $\mathbf{S}^{n}$ (endowed with its canonical Riemannian metric of curvature +1$)$. Any lightlike geodesic is left invariant by the action of the center (so that lightlike geodesics on $\mathbf{E i n}_{n+1}$ are circles).

Given a point $p$ in $\widetilde{\mathbf{E i n}}_{n+1}$, the lightcone with vertex $p$, denoted by $C(p)$, is the set of lightlike geodesics passing through $p$. Lightcones are not smooth submanifolds of $\widetilde{\operatorname{Ein}}_{n+1}$. The singular points are exactly the points $\zeta^{k} . p$, for $k \in \mathbb{Z}$. Removing its singular points to a lightcone, one gets a countably infinite family of connected components, each one diffeomorphic to the product $\mathbf{R} \times \mathbf{S}^{n-1}$.

\subsection{The conformal boundary completion of $\widetilde{\operatorname{AdS}}_{n+1}$}

In $\mathbf{R}^{2, n+1}$, we denote by $\mathbf{R}^{2, n}$ the subspace generated by $e_{1}, \ldots, e_{n}, e_{n+2}, e_{n+3}$. The projection of $\mathbf{R}^{2, n} \cap C^{2, n+1}$ on $\mathbf{E i n}_{n+1}$ is a sub-Einstein's universe of codimension 1, that we call $\mathbf{E i n}_{n}$. The complementary of $\mathbf{E i n}_{n}$ in $\mathbf{E i n}_{n+1}$ is exactly the projection of the set $Q=\left\{u+e_{n+1} \in \mathbf{R}^{2, n+1} \mid u \in \mathbf{R}^{2, n}, q^{2, n+1}(u)=\right.$ $-1\}$. It is, conformally, the space $\mathbf{A d} \mathbf{S}_{n+1}$ (namely, the quotient of the antide Sitter space $\widetilde{\mathbf{A d S}}_{n+1}$ by the center of its isometry group). The subspace $\operatorname{Ein}_{n}$ lift into a subspace $\widetilde{\operatorname{Ein}}_{n} \subset \widetilde{\operatorname{Ein}}_{n+1}$. In this model, the complementary of $\widetilde{\mathbf{E i n}}_{n}$ in $\widetilde{\operatorname{Ein}}_{n+1}$ has two connected components, each one conformally equivalent to $\widetilde{\mathbf{A d S}}_{n+1}$. We chose one of these components, that we call $\widetilde{\mathbf{A d S}}_{n+1}$. The union $\widehat{\mathbf{A d S}}_{n+1} \cup \widetilde{\mathbf{E i n}}_{n}=\overline{\mathbf{A d S}}_{n+1}$ is a submanifold of $\widetilde{\operatorname{Ein}}_{n+1}$, with boundary $\widetilde{\operatorname{Ein}}_{n}$, and interior $\widetilde{\mathbf{A d S}}_{n+1}$. The canonical conformal structure on $\widetilde{\operatorname{Ein}}_{n+1}$ induces a canonical conformal structure $\left[\bar{g}_{c a n}\right]$ on $\overline{\mathbf{A d S}}_{n+1}$, and $\left(\overline{\mathbf{A d S}}_{n+1},\left[\bar{g}_{c a n}\right]\right)$ is a conformal boundary completion of $\widetilde{\operatorname{AdS}}_{n+1}$.

The subgroup $\widetilde{O(2, n)} \subset O\left(\widetilde{2, n+1)}\right.$ which leaves $\mathbf{R}^{2, n} \subset \mathbf{R}^{2, n+1}$ invariant, acts isometrically on $\widetilde{\mathbf{A d S}}_{n+1}$, and conformally on $\overline{\mathbf{A d S}}_{n+1}$. 


\section{Conformal dynamics}

\subsection{Cartan's decomposition}

The group $\widetilde{O(2, n)}$ is not a matrix group, and it is not so easy to understand its dynamics on $\widetilde{\mathbf{E i n}}_{n}$, or $\overline{\mathbf{A d S}}_{n+1}$. Nevertheless, some specific subgroups or subsets of $\widetilde{O(2, n)}$ are simple to describe, and help in the understanding of the whole group:

- The center of $\widetilde{S O^{o}(2, n)}$, isomorphic to $\mathbb{Z}$, and generated by the transformation $\zeta:(t, x) \mapsto(t+\pi,-x)$ (we denote by $(t, x)$ the points of Einstein's universe $\left.\mathbf{R} \times \mathbf{S}^{n-1}\right)$.

- The subset $K$ consisting of transformations of the form: $(t, x) \mapsto$ $(t+a, \sigma \cdot x)$, with $a \in[0, \pi[$ and $\sigma \in O(n-2)$. Note that $K$ is relatively compact.

- The abelian subgroup $A^{+}$, which projects injectively in $O(2, n)$ onto the subgroup of matrices:

$$
\left(\begin{array}{ccccccc}
e^{\lambda} & & & & & & \\
& e^{\mu} & & & & & \\
& & 1 & & & & \\
& & & \ddots & & & \\
& & & 1 & & \\
& & & & e^{-\mu} & \\
& & & & & & e^{-\lambda}
\end{array}\right)
$$

with $\lambda \geq \mu \geq 0$.

Now, every element $g$ of $\widetilde{O(2, n)}$ can be written $g=\zeta^{l(g)} k_{1}(g) a^{+}(g) k_{2}(g)$, with $l(g) \in \mathbb{Z}, k_{1}(g)$ and $k_{2}(g)$ in $K$, and $a^{+}(g) \in A^{+}$. This decomposition is called Cartan's decomposition of $\widetilde{O(2, n)}$. Notice that the integer $l(g)$ and the Cartan's projection $a^{+}(g)$ are uniquely determined by $g$.

Let us write $a^{+}(g)$ as a matrix of $O(2, n)$ :

$$
a^{+}(g)=\left(\begin{array}{ccccccc}
e^{\lambda(g)} & & & & & & \\
& e^{\mu(g)} & & & & & \\
& & 1 & & & & \\
& & & \ddots & & & \\
& & & 1 & & \\
& & & & & e^{-\mu(g)} & \\
& & & & & & e^{-\lambda(g)}
\end{array}\right)
$$

The reals $\lambda(g) \geq \mu(g) \geq 0$ are called the distorsions of the element $g$. 


\subsection{Sequences tending to infinity}

Let us consider now a sequence $\left(g_{k}\right)$ in $\widetilde{O(2, n)}$, which tends to infinity (i.e leaves every compact subset of $\widehat{O(2, n)})$. Looking, if necessary, at a subsequence, we can suppose:

a) the four sequences $l\left(g_{k}\right), \lambda\left(g_{k}\right), \mu\left(g_{k}\right)$ and $\delta\left(g_{k}\right)=\lambda\left(g_{k}\right)-\mu\left(g_{k}\right)$ converge respectively to $l_{\infty}, \lambda_{\infty}, \mu_{\infty}$ and $\delta_{\infty}$ in $\overline{\mathbf{R}}$.

b) compact factors in the Cartan's decomposition of $\left(g_{k}\right)$ both admit a limit in $\bar{K}$, as $k$ tends to infinity.

Under these asumptions, the sequence $\left(g_{k}\right)$ falls into one of the four following categories:

(i) $\left|l_{\infty}\right|=+\infty$. The sequence $\left(g_{k}\right)$ is then said to be proper.

For the three other cases, $l_{\infty}$ is finite.

(ii) If $\mu_{\infty} \neq+\infty$, the sequence $\left(g_{k}\right)$ is said to have bounded distorsion.

(iii) If $\lambda_{\infty}=\mu_{\infty}=+\infty$ and $\delta_{\infty}$ is finite, the sequence $\left(g_{k}\right)$ is said to have balanced distorsions.

(iv) If $\lambda_{\infty}=\mu_{\infty}=\delta_{\infty}=+\infty$, the sequence $\left(g_{k}\right)$ is said to have mixed distorsions.

\subsection{The limit set of a complete anti-de Sitter structure}

In [Fr2], we studied extensively the dynamical behaviour on $\operatorname{Ein}_{n}$ of sequences tending to infinity in $O(2, n)$. This description can be adapted to sequences of $\widetilde{O(2, n}$ ) acting on $\overline{\mathbf{A d S}}_{n+1}$ (resp. $\widetilde{\mathbf{E i n}}_{n}$ ), and we refer to [Fr2] for the proofs. Here, we state only the few properties which will be relevant for our purpose. First, we recall the following definition:

Definition 1 (Properness). Let $\left(g_{k}\right)$ be a sequence of $\widetilde{O(2, n)}$ tending to infinity, and $\Omega$ an open subset of $\overline{\mathbf{A d S}}_{n+1}$, left invariant by $\left(g_{k}\right)$. The sequence $\left(g_{k}\right)$ acts properly on $\Omega$ if for any pair $\left(K, K^{\prime}\right)$ of compact subsets of $\Omega, g_{k}(K) \cap K^{\prime}=\emptyset$ for all but a finite number of $k$ 's.

Proposition 1. A sequence $\left(g_{k}\right)$ of $\widetilde{O(2, n)}$ tending to infinity acts properly on $\overline{\mathbf{A d S}}_{n+1}$ if and only if it is proper.

In Riemannian signature, Ascoli's theorem ensures that a sequence of isometries of a manifold $X$ always acts properly on $X$. This is no longer true in Lorentzian geometry, and this is what makes Lorentzian dynamics richer. For example, a sequence of $\widetilde{O(2, n)}$ can act non properly on $\widetilde{\mathbf{A d S}}_{n+1}$. The next proposition is a usefull caraterisation of sequences in $\widetilde{O(2, n)}$ acting properly on $\widetilde{\mathbf{A d S}}_{n+1}$.

Proposition 2. A sequence $\left(g_{k}\right)$ of $\widetilde{O(2, n)}$ acts properly on $\widetilde{\mathbf{A d S}}_{n+1}$ if and only if $\left(g_{k}\right)$ does not have any subsequence with bounded distortion. In 
particular, a discrete subgroup $\Gamma \subset \widetilde{O(2, n)}$ acts properly discontinuously on $\widetilde{\mathbf{A d S}}_{n+1}$ if and only if $\Gamma$ does not contain any infinite sequence with bounded distorsion.

The next step is the dynamical description of sequences with mixed or balanced distorsions.

Proposition 3. Let $\left(g_{k}\right)$ be an infinite sequence of $\widetilde{O(2, n)}$, with bounded or mixed distorsions. There are two lightlike geodesics $\Delta^{+}\left(g_{k}\right)$ and $\Delta^{-}\left(g_{k}\right)$ in $\widetilde{\operatorname{Ein}}_{n}$, called attracting and repelling geodesics, uniquely determined by the sequence $\left(g_{k}\right)$, such that:

For any compact subset $K \subset \overline{\mathbf{A d S}}_{n+1} \backslash\left\{\Delta^{-}\left(g_{k}\right)\right\}$ (resp. $\left.K \subset \overline{\mathbf{A d S}}_{n+1} \backslash\left\{\Delta^{+}\left(g_{k}\right)\right\}\right)$, $g_{k}(K)$ (resp. $\left.g_{k}^{-1}(K)\right)$ tends (for the Hausdorff topology) to a closed subset of $\Delta^{+}\left(g_{k}\right)$ (resp. $\left.\Delta^{-}\left(g_{k}\right)\right)$.

Let $M$ be a manifold endowed with a complete anti-de Sitter metric $g$. The structure $(M, g)$ is obtained as the quotient of the space $\widetilde{\mathbf{A d S}}_{n+1}$, by a discrete subgroup $\Gamma \subset \widetilde{O(2, n)}$, acting properly discontinuously on $\widetilde{\mathbf{A d S}}_{n+1}$. By Proposition 2, the group $\Gamma$ does not contain any sequence $\left(\gamma_{k}\right)$ with bounded distorsion. Let $\mathcal{S}$ be the set of all sequences $\left(\gamma_{k}\right) \subset \Gamma$, which have either mixed or balanced distorsion. Notice that any infinite sequence of $\Gamma$ has a subsequence in $\mathcal{S}$. We define the limit set of the group $\Gamma$ in the following way:

$$
\Lambda_{\Gamma}=\overline{\bigcup_{\left(\gamma_{k}\right) \in \mathcal{S}} \Delta^{+}\left(\gamma_{k}\right) \cup \Delta^{-}\left(\gamma_{k}\right)}
$$

It is clear that the set $\Lambda_{\Gamma}$ is closed, $\Gamma$-invariant, and included in $\widetilde{\mathbf{E i n}_{n}}$. Let us call $\Omega_{\Gamma}$ the complementary of $\Lambda_{\Gamma}$ in $\overline{\mathbf{A d S}}_{n+1}$. We deduce easily from Proposition 3:

Proposition 4. The action of $\Gamma$ on $\Omega_{\Gamma}$ is proper and discontinuous.

For a more general definition of the limit set of a discrete subgroup $\Gamma \subset O(2, n)$, we refer to [Fr2]. Other definitions of the limit set, in the framework of linear discrete groups can be found in $[\mathrm{B}]$.

\section{The conformal boundary completion of a com- plete anti-de Sitter structure}

\subsection{Building the boundary}

Let $(M, g)$ be a complete anti-de Sitter structure, obtained as the quotient $\widetilde{\mathbf{A d S}}_{n+1} / \Gamma$. By proposition 4 , the quotient $\bar{M}=\Omega_{\Gamma} / \Gamma$ is a manifold 
with boundary. The interior of $\bar{M}$ is the complete anti-de Sitter structure $\widetilde{\mathbf{A d S}}_{n+1} / \Gamma$ we started with. The boundary $\partial M$ is the conformally flat Lorentzian manifold $\left(\Omega_{\Gamma} \cap \widetilde{\operatorname{Ein}}_{n}\right) / \Gamma$. In fact, $\bar{M}$ inherits from $\Omega_{\Gamma}$ a canonical conformal class of Lorentzian metrics. Let us pick some metric $\bar{g}$ in this class. We lift $\bar{g}$ (resp. $g$ ) into a $\Gamma$-invariant $\bar{h}$ (resp. $h$ ) on $\Omega_{\Gamma}\left(\right.$ resp. $\left.\widetilde{\mathbf{A d S}}_{n+1}\right)$. The metric $h$ is nothing else than the canonical metric $g_{\text {can }}$ of $\widetilde{\mathbf{A d S}}_{n+1}$. For this canonical metric, there is a well known conformal boundary. So, there is a smooth metric $\bar{h}^{\prime}$ on $\overline{\mathbf{A d S}}_{n+1}$, and a function $\beta$ on $\widehat{\mathbf{A d S}}_{n+1}$, such that $\bar{h}^{\prime}=\beta^{2} h$. Moreover, $\widehat{\operatorname{Ein}}_{n}=\beta^{-1}(\{0\})$ and $d \beta \neq 0$ on $\widehat{\operatorname{Ein}}_{n}$. On the other hand, $\bar{h}$ and $\bar{h}^{\prime}$ are in the same conformal class, so that $\bar{h}=\alpha^{2} \bar{h}^{\prime}$ on $\Omega_{\Gamma}$. We thus get $\bar{h}=\rho^{2} h$ on $\widetilde{\mathbf{A d S}}_{n+1}$, with $\rho=\alpha \beta$. The function $\rho$ is smooth on $\Omega_{\Gamma}, \rho^{-1}(\{0\})=\Omega_{\Gamma} \cap \widetilde{\operatorname{Ein}}_{n}$ and $d \rho \neq 0$ on $\Omega_{\Gamma} \cap{\widetilde{\operatorname{Ein}_{n}}}_{n}$. Moreover, it has to be $\Gamma$-invariant, since it is the case for $\bar{h}$ and $h$. Thus, $\rho$ induces a smooth function $\bar{\rho}$ on $\bar{M}$, such that $\bar{g}=\bar{\rho}^{2} g$ on $M$. Clearly $\bar{\rho}^{-1}(\{0\})=\partial M$ and $d \bar{\rho} \neq 0$ on $\partial M$. This proves that $\bar{M}$ is a conformal boundary completion of $(M, g)$.

Remark 1. Of course, it can happen that for some complete structure $\widetilde{\mathbf{A d S}}_{n+1} / \Gamma, \Lambda_{\Gamma}=\widetilde{\mathbf{E i n}}_{n}$. In this case, the previous construction yields an empty conformal boundary $\partial M$.

\section{$4.2(G, X, \partial X)$-structures}

Let $\bar{X}$ be a manifold with boundary $\partial X$, and interior $X$. We write $\bar{X}=$ $X \cup \partial X$. Suppose that some Lie group $G$ acts transitively on $X \cup \partial X$. Suppose moreover that the action of $G$ is strongly effective. It means that two elements $g_{1}$ and $g_{2}$ of $G$ acting in the same way on an open subset $U \subset X$ are in fact equal. Then we can define the notion of a $(G, X, \partial X)$-structure on a manifold $\bar{M}$, with boundary $\partial M$ and interior $M$. It is the the data of:

- an open covering $\left(U_{i}\right)_{i \in I}$ of $\bar{M}$.

- a family of embeddings $\phi_{i}: U_{i} \rightarrow \bar{X}$, respecting the boundaries (i.e $\left.\phi_{i}\left(U_{i} \cap \partial M\right)=\phi_{i}\left(U_{i}\right) \cap \partial X\right)$.

- a family $\left(g_{j i}\right)_{i, j \in I}$ of elements of $G$, such that for all $i, j \in I$, the map $\phi_{j} \circ \phi_{i}^{-1}$ agrees with $g_{j i}$ on $\phi_{i}\left(U_{i} \cap U_{j}\right)$.

With this definition, the conformal boundary completion of a complete anti-de Sitter structure, as defined above, is endowed with a $\left(\widetilde{O(2, n)}, \widetilde{\mathbf{A d S}}+\widetilde{\mathbf{E i n}}_{n+1}\right)$ structure.

\subsection{Main properties of the conformal boundary completion for a complete anti-de Sitter structure}

The first, and maybe most important property, is that, under some natural asumptions, there is essentially a unique conformal boundary completion, for 
a given complete anti-de Sitter structure. Since we are dealing, a priori, with non compact conformal boundary completions, it is natural to introduce the notion of maximal completion.

Definition 2. Let $(\bar{M},[\bar{g}])$ and $(\bar{N},[\bar{h}])$ two conformal boundary completions of a space-time $(M, g)$. We write $(\bar{M},[\bar{g}]) \subset(\bar{N},[\bar{h}])$ if there is a conformal embedding of $(\bar{M},[\bar{g}])$ into $(\bar{N},[\bar{h}])$, mapping $\partial M$ into $\partial N$. A maximal element for the relation $\subset$ is called a maximal conformal boundary completion of $(M, g)$.

We can now prove:

Theorem 1. Let $(M, g)$ a complete anti-de Sitter structure. If $(\bar{M},[\bar{g}])$ and $(\bar{N},[\bar{h}])$ are two maximal conformal boundary completions of $M$, and if moreover $\bar{M}$ and $\bar{N}$ both admit a $\left(\widetilde{O(2, n)}, \widetilde{\mathbf{A d S}}_{n+1}, \widetilde{\operatorname{Ein}}_{n}\right)$-structure, then they are isomorphic (i.e there is a diffeomorphism of manifolds with boundary between $\bar{M}$ and $\bar{N}$, which is conformal).

Proof: We write $\bar{M}=M \cup \partial M$ and denote by $\widetilde{M \cup \partial M}$ the universal cover of $\bar{M}$. As for classical $(G, X)$-structures, $(G, X, \partial X)$-structures admit a developping map $\delta$ and a holonomy morphism $\rho$ (see [Th]). In our case $\delta$ is a local diffeomorphism from $\widetilde{M \cup \partial M}$ to $\overline{\mathbf{A d S}}_{n+1}$, which sends the boundary of $\widetilde{M \cup \partial M}$ in $\widetilde{\operatorname{Ein}}_{n}$. The interior of $\widetilde{M \cup \partial M}$ is just $\tilde{M}$, the universal cover of $M$. The restriction of $\delta$ to $\tilde{M}$ is the developping map of the anti-de Sitter structure on $M$. Since $(M, g)$ is complete, $\delta_{\mid \tilde{M}}$ is injective. Since $\delta$ is a local diffeomorphism, we infer that $\delta$ is injective on the whole $\widetilde{M \cup \partial M}$. Thus, $(\bar{M},[\bar{g}])$ is obtained as a quotient $\Omega / \Gamma$, where $\Omega$ is an open subset of $\overline{\mathbf{A d S}}_{n+1}$ and $\Gamma$ is the discrete group of $\widetilde{O(2, n)}$, such that $(M, g)=\widetilde{\mathbf{A d S}}_{n+1} / \Gamma$. We now prove the:

Lemma 1. If a discrete subgroup $\Gamma \subset \widetilde{O(2, n)}$ acts properly on some open subset $\Omega$, with $\widetilde{\mathbf{A d S}}_{n+1} \subset \Omega \subset \overline{\mathbf{A d S}}_{n+1}$, then $\Omega \subset \Omega_{\Gamma}$.

Proof: Since $\widetilde{\mathbf{A d S}}_{n+1} \subset \Omega$ and $\Gamma$ acts properly on $\Omega$, we know from section 3.3 that $\Gamma$ has a limit set $\Lambda_{\Gamma}$, and so $\Omega_{\Gamma}$ is well defined. Now, let $\left(\gamma_{k}\right)$ be a sequence of $\Gamma$ with balanced or mixed distorsions. We call $\Delta^{+}\left(\gamma_{k}\right)$ and $\Delta^{-}\left(\gamma_{k}\right)$ its attracting and repelling lightlike geodesics. We project $\left(\gamma_{k}\right)$ on $\bar{\gamma}_{k} \in O(2, n)$ and $\Delta^{+}\left(\gamma_{k}\right)\left(\right.$ resp. $\left.\Delta^{-}\left(\gamma_{k}\right)\right)$ on $\bar{\Delta}^{+} \subset \operatorname{Ein}_{n}\left(\right.$ resp. $\left.\bar{\Delta}^{-} \subset \operatorname{Ein}_{n}\right)$. Let $x \in \Delta^{-}$, and a $U$ be a small neighbourhood of $x$ in $\operatorname{Ein}_{n+1}$. in [Fr2] (sections 3.2.1, 3.2.3), we proved that the Hausdorff $\operatorname{limit}_{k \rightarrow+\infty} \lim _{k}(U)$ is either a lightcone (of $\mathbf{E i n}_{n+1}$ ), or some closed subset with non empty interior. In any case, this limit meets the complementary of $\mathbf{E i n}_{n}$ in $\mathbf{E i n}_{n+1}$. Lifting all these results to $\widetilde{\mathbf{E i n}}_{n}$, we get that if $x \in \Delta^{-}\left(\gamma_{k}\right)$, and $U$ is a small neighbourhood of $x$ in $\overline{\mathbf{A d S}}_{n+1}$, then the Hausdorff limit of $\bar{\gamma}_{k}(U)$ meets 
$\widetilde{\mathbf{A d S}}_{n+1}$. So, if $\Omega$ meets $\Delta^{-}\left(\gamma_{k}\right)$, the action can't be proper on $\Omega$, since $\widetilde{\mathbf{A d S}}_{n+1} \subset \Omega$ by hypothesis. Looking at $\left(\gamma_{k}^{-1}\right)$, one proves also that $\Omega$ can't meet $\Delta^{+}\left(\gamma_{k}\right)$. We infer that $\Lambda_{\Gamma} \cap \Omega=\emptyset$, and $\Omega \subset \Omega_{\Gamma}$.

The previous result and the maximality hypothesis on $(\bar{M},[\bar{g}])$ yields that $(\bar{M},[\bar{g}])=\Omega_{\Gamma} / \Gamma$. So the structure of $(\bar{M},[\bar{g}])$ is entirely defined under the two hypothesis that $(\bar{M},[\bar{g}])$ is maximal and shares a $\left(\widetilde{O(2, n)}, \widetilde{\mathbf{A d S}}_{n+1}, \widetilde{\left.\mathbf{E i n}_{n}\right)}\right.$ structure.

From now, when we will speak of the conformal boundary completion of a complete anti-de Sitter structure $(M, g)=\widetilde{\mathbf{A d S}}_{n+1} / \Gamma$, we will always mean $(\bar{M},[\bar{g}])=\Omega_{\Gamma} / \Gamma$ (this is justified by Theorem 1$)$.

Theorem 2. Let $(M, g)=\widetilde{\mathbf{A d S}}_{n+1} / \Gamma_{1}$ and $(N, h)=\widetilde{\mathbf{A d S}}_{n+1} / \Gamma_{2}$ be two complete anti-de Sitter structures of dimension $\geq 4$, with conformal boundary completions $(\bar{M},[\bar{g}])$ and $(\bar{N},[\bar{h}])$. Suppose that the manifolds $\left(\partial M,[\bar{g}]_{\mid \partial M}\right)$ and $\left(\partial N,[\bar{h}]_{\mid \partial N}\right)$ are conformally equivalent, then $(M, g)$ and $(N, h)$ are isometric.

Proof: The structures $\left(\partial M,[\bar{g}]_{\mid \partial M}\right)$ and $\left(\partial N,[\bar{h}]_{\mid \partial N}\right)$ are Kleinian, obtained as quotients $\left(\Omega_{\Gamma_{1}} \cap \widetilde{\operatorname{Ein}}_{n}\right) / \Gamma_{1}$ and $\left(\Omega_{\Gamma_{2}} \cap \widetilde{\operatorname{Ein}_{n}}\right) / \Gamma_{2}$ respectively. A conformal diffeomorphism $\bar{\phi}$ from $\left(\partial M,[\bar{g}]_{\mid \partial M}\right)$ to $\left(\partial N,[\bar{h}]_{\mid \partial N}\right)$ yields a conformal transformation $\phi$ between $\Omega_{\Gamma_{1}} \cap \widetilde{\operatorname{Ein}}_{n}$ and $\Omega_{\Gamma_{2}} \cap \widetilde{\operatorname{Ein}}_{n}$ such that $\phi \Gamma_{1}=\Gamma_{2} \phi$. But in dimension $\geq 3$, any conformal transformation between $\Omega_{1}$ and $\Omega_{2}$ is the restriction of some element of $\widetilde{O(2, n)}$, by Liouville's theorem (see $[\mathrm{S}]$, [Fr3]). So $\phi \in \widetilde{O(2, n)}$ and $\phi \Gamma_{1} \phi^{-1}=\Gamma_{2}$. Thus, since $(M, g)$ and $(N, h)$ are respectively $\widetilde{\mathbf{A d S}}_{n+1} / \Gamma_{1}$ and $\widetilde{\mathbf{A d S}}_{n+1} / \Gamma_{2},(M, g)$ and $(N, h)$ are isometric.

Proposition 5. Let $(M, g)$ be a complete anti-de Sitter structure, and $(\bar{M},[\bar{g}])$ its conformal boundary completion. Then any isometry of $(M, g)$ extends to a diffeomorphism of $\bar{M}$, acting conformally on $\left(\partial M,[\bar{g}]_{\mid \partial M}\right)$. Reciprocally, any conformal transformation of $\left(\partial M,[\bar{g}]_{\mid \partial N}\right)$ extends to a diffeomorphism of $\bar{M}$, acting isometrically on $(M, g)$.

Proof: If we write $(M, g)$ as a quotient $\widetilde{\mathbf{A d S}}_{n+1} / \Gamma$, the structure $\left(\partial M,[\bar{g}]_{\mid \partial M}\right)$ is a Kleinian structure, obtained as the quotient $\left(\Omega_{\Gamma} \cap \widetilde{\mathbf{E i n}}_{n}\right) / \Gamma$. Any isometry of $(M, g)$ is induced by an element $\phi \in \widetilde{O(2, n)}$ satisfying $\phi \Gamma \phi^{-1}=\Gamma$. But since $\phi\left(\Lambda_{\Gamma}\right)=\Lambda_{\phi \Gamma \phi^{-1}}$, we get that $\phi\left(\Omega_{\Gamma}\right)=\Omega_{\Gamma}$, and $\phi$ induces a conformal diffeomorphism of $(\bar{M},[\bar{g}])$. In particular, it induces a conformal diffeomorphism of $\left(\partial M,[\bar{g}]_{\mid \partial M}\right)$. Reciprocally, a conformal transformation 
of $\left(\partial M,[\bar{g}]_{\mid \partial M}\right)$ is induced by a $\phi \in \widetilde{O(2, n)}$, such that $\phi \Gamma \phi^{-1}=\Gamma$ and $\phi\left(\Omega_{\Gamma} \cap \widetilde{\operatorname{Ein}}_{n}\right)=\Omega_{\Gamma} \cap \widetilde{\operatorname{Ein}}_{n}$. But then, $\phi\left(\Omega_{\Gamma}\right)=\Omega_{\Gamma}$ and $\phi$ induces a diffeomorphism of $\bar{M}$. This diffeomorphism is moreover an isometry of $(M, g)$, since $\phi\left(\widetilde{\mathbf{A d S}}_{n+1}\right)=\widetilde{\mathbf{A d S}}_{n+1}$ and $\phi \Gamma \phi^{-1}=\Gamma$.

\section{Illustration}

\subsection{The example of Lorentzian Schottky groups}

Let $\Delta_{i}^{ \pm}, i=1, \ldots, g$, be a collection of $2 g$ pairwise disjoint lightlike geodesics in $\widetilde{\operatorname{Ein}}_{n}$. Then there exist $g$ elements $\gamma_{1}, \ldots, \gamma_{g}$ in $\widetilde{O(2, n)}$, and a family $U_{i}^{ \pm}$ of $2 g$ open subsets of $\overline{\mathbf{A d S}}_{n+1}$, with the following properties:

(i) For all $1 \leq i \leq g, U_{i}^{ \pm}$is a tubular neighbourhood of $\Delta_{i}^{ \pm}$.

(ii) The closures of the $U_{i}^{ \pm}$'s are pairwise disjoint.

(iii) For all $1 \leq i \leq g, \gamma_{i}$ (resp. $\gamma_{i}^{-1}$ ) maps the exterior of $U_{i}^{-}$(resp. $U_{i}^{+}$) in $\overline{\mathbf{A d S}}_{n+1}$, on the closure of $U_{i}^{+}$(resp. $U_{i}^{-}$).

The group $\Gamma$ generated by the elements $\gamma_{1}, \ldots, \gamma_{g}$ is then a free group, called Lorentzian Schottky group. We studied such groups in [Fr2]. In particular, we proved:

Theorem 3. Let $\Gamma \subset \widetilde{O(2, n)}(n \geq 3)$, be a Lorentzian Schottky group with $g$ generators $(g \geq 2)$. Then:

(i) The action of $\Gamma$ on $\widetilde{\mathbf{A d S}}_{n+1}$ is proper.

(ii) The limit set $\Lambda_{\Gamma}$ is a lamination by lightlike geodesics. Topologically, it is a product of $\mathbf{R}$ by a Cantor set.

(iii) The conformal boundary of the complete anti-de Sitter space-time $\widetilde{\mathbf{A d S}}_{n+1} / \Gamma$ is diffeomorphic to $\mathbf{R} \times\left(\mathbf{S}^{1} \times \mathbf{S}^{n-2}\right)^{\sharp(g-1)}$. Here, $\left(\mathbf{S}^{1} \times \mathbf{S}^{n-2}\right)^{\sharp(g-1)}$ denotes the connected sum of $g-1$ copies of $\left(\mathbf{S}^{1} \times \mathbf{S}^{n-2}\right)$.

For $n=2$, there is an alternative statement to this theorem, but this time, the conformal boundary is not connected: it is a finite union of cylin$\operatorname{ders} \mathbf{R} \times \mathbf{S}^{1}$.

\subsection{Other examples in odd dimension}

The group $U(1, n)$, preserving the Lorentzian hermitian form $-\left|z_{1}\right|^{2}+\left|z_{2}\right|^{2}+$ $\ldots+\left|z_{n}\right|^{2}$, admits a canonical injection in $O(2,2 n)$. This yields an injection of the universal covers $\widetilde{U(1, n)} \subset \widetilde{O(2,2 n)}$. In fact, there is a natural fibration of $\widetilde{\mathbf{E i n}}_{2 n}$ over the canonical flat $C R$-sphere $\mathbf{S}^{2 n-1}$, the fibers of which are lightlike geodesics. The group $\widetilde{U(1, n)}$ is exactly the subgroup of $\widetilde{O(2,2 n)}$ preserving this fibration. On the other hand, as first observed by R. Kulkarni, $\widetilde{U(1, n)}$ acts properly on $\widetilde{\mathbf{A d S}}_{2 n+1}$. Let $(M, g)$ be a complete anti-de Sitter structure, obtained as a quotient $\mathbf{A d S}_{2 n+1} / \Gamma$, with $\Gamma \subset \widetilde{U(1, n)}$. The 
group $\Gamma$ projects as a subgroup $\bar{\Gamma} \subset U(1, n)$. The group $\bar{\Gamma}$ acts on $\mathbf{S}^{2 n-1}$ as a convergence group. In particular, it has a domain of discontinuity $\bar{\Omega}_{\bar{\Gamma}}$ (which turns out to be the projection of $\Omega_{\Gamma} \cap \mathbf{E i n}_{2 n}$ ). We obtain that the conformal boundary of $(M, g)$ is a fibration by lightlike geodesics over the $C R$-flat Kleinian manifold $\bar{\Omega}_{\bar{\Gamma}} / \bar{G}$ (we refer to [Go] for an account on $C R$ flat Kleinian manifolds). In other words, the structure $(M, g)$ fibers over a complete complex hyperbolic manifold, the boundary of which is the $C R$ manifold $\bar{\Omega}_{\bar{\Gamma}} / \bar{\Gamma}$. We just saw that the fibration extends to the respective boundaries.

Aknowledgments: I would like to thank A.Zeghib for usefull comments on this text.

\section{References}

[A] M.ANDERSON - Einstein metrics with prescribed conformal infinity on 4-Manifolds. Preprint. arXiv:math.DG/0105243

[B] Y.Benoist - Propriétés asymptotiques des groupes linéaires. Geom. Funct. Anal. 7 (1997), no. 1, 1-47.

[Bi] O.BIquARD - Métriques d"Einstein asymptotiquement symétriques. Astérisque no 265. (2000)

[BTZ1] M.Bañados; C.Teitelboim; J.ZAnelli - Black hole in threedimensional space-time. Phys. Rev. Lett. 69 (1992), no. 13, 18491851

[BTZ2] M.Bañados; M.Henneaux; C.Teitelboim; J.Zanelli - Geometry of the $2+1$ black hole. Phys. Rev. D (3) 48 (1993), no. $4,1506-1525$.

[CK] M.CAhen; Y.Kerbrat - Domaines symétriques des quadriques projectives. J. Math. Pures Appl. (9) 62 (1983), no. 3, 327-348.

[C-H] P.Chrusciel, M.Herzlich - The mass of asymptotically hyperbolic Riemannian manifolds. Pacific J. Math. 212 (2003), no. 2, 231-264.

[Fr1] C.Frances - Géométrie et dynamique lorentziennes conformes. Thèse. ENS-Lyon (2002). Available at http://www.umpa.enslyon.fr/ cfrances/.

[Fr2] C.FrAnCES - Lorentzian Kleinian groups. preprint (2003). Available at $h t t p: / / w w w . u m p a . e n s-l y o n . f r / \sim c f r a n c e s /$. 
[Fr3] C. FRANCES - Les structures conformes pseudo-riemanniennes vues comme structures rigides à l'ordre 2. Preprint (2002). Available at http://www.umpa.ens-lyon.fr/ cfrances/.

[Go] W.Goldman - Complex hyperbolic kleinian groups. in: Complex geometry, Proceedings of the Osaka International Conference, Marcel Dekker, Inc., New York, G. Komatsu and Y. Sakane (Eds.) (1992), 31-52.

[HE] S.Hawking; G.ElLis - The large scale structure of universeCambridge University Press, 1973.

[IW] A.IozzI; D.WITTE - Cartan-decomposition subgroups of $\mathrm{SU}(2, n)$. J. Lie Theory 11 (2001), no. 2, 505-543.

[O'N] B.O'NeILL - Semi-riemannian geometry with applications to relativity- New York, Academic Press Inc, 1983.

[P] R.Penrose - Conformal treatment of infinity. in Relativit, Groupes et Topologie (Lectures, Les Houches, 1963 Summer School of Theoret. Phys., Univ. Grenoble) pp. 563-584 Gordon and Breach, New Ann. Global Anal. Geom. 21 (2002), no. 1, 5162 York.

[S] I. Segal, Mathematical cosmology and extragalactic astronomy. Pure and Applied Mathematics, Vol. 68. Academic Press [Harcourt Brace Jovanovich, Publishers], New York-London, 1976.

[Th] W.Thurston - Three dimensional geometry and topology. Vol 1.Princeton University Press, 1997. Edited by Silvio Levy.

[Wo] J.Wolf - Spaces of constant curvature - Houston, Tex., Publish or Perish Inc., 1984, fifth edition.

[Ze] A.ZeGHib - On closed anti de Sitter space-times- Math.Ann. 310 695-716 (1998)

\section{Charles FRANCES}

ENS Lyon

46 allée d'Italie

69364 Lyon cedex 07

email: cfrances@umpa.ens-lyon.fr 